TAMKANG JOURNAL OF MATHEMATICS

Volume 33, Number 3, Autumn 2002

\title{
A NOTE ON DRAGOMIR-MCANDREW'S TRAPEZOID INEQUALITIES
}

\author{
HENDRA GUNAWAN
}

\begin{abstract}
In [1], Dragomir and Mcandrew proved some trapezoid inequalities by involving a Grüss' type inequality. In this note, we show that their trapezoid inequalities can actually be obtained directly in a much simpler way. Some improvements will also be offered.
\end{abstract}

\section{Introduction. A Direct Proof of Dragomir-Mcandrew's Results}

Let $f, g:[a, b] \rightarrow \mathbf{R}$ be integrable. Write $\int_{a}^{b} f$ for $\int_{a}^{b} f(x) d x$. Then, Dragomir and Mcandrew [1] showed that the following Grüss' type inequality

$\left|\frac{1}{b-a} \int_{a}^{b} f g-\left[\frac{1}{b-a} \int_{a}^{b} f\right]\left[\frac{1}{b-a} \int_{a}^{b} g\right]\right| \leq \frac{1}{b-a} \int_{a}^{b}\left|\left[f(x)-\frac{1}{b-a} \int_{a}^{b} f\right]\left[g(x)-\frac{1}{b-a} \int_{a}^{b} g\right]\right| d x(1)$

holds. Moreover, by taking $f(x)=g(x)=\operatorname{sign}\left(x-\frac{a+b}{2}\right)$, they confirmed that the inequality is sharp.

From (1), they then derived the following trapezoid inequalities for functions $f$ having first derivative bounded, integrable, or $q$-integrable on $(a, b)$ :

$$
\begin{aligned}
& \left|\frac{f(a)+f(b)}{2}-\frac{1}{b-a} \int_{a}^{b} f\right| \leq \frac{b-a}{4} \sup _{x \in(a, b)}\left|f^{\prime}(x)-\frac{f(b)-f(a)}{b-a}\right| \\
& \left|\frac{f(a)+f(b)}{2}-\frac{1}{b-a} \int_{a}^{b} f\right| \leq \frac{1}{2} \int_{a}^{b}\left|f^{\prime}(x)-\frac{f(b)-f(a)}{b-a}\right| d x \\
& \left|\frac{f(a)+f(b)}{2}-\frac{1}{b-a} \int_{a}^{b} f\right| \leq \frac{1}{2}\left[\frac{b-a}{p+1}\right]^{1 / p}\left[\int_{a}^{b}\left|f^{\prime}(x)-\frac{f(b)-f(a)}{b-a}\right|^{q} d x\right]^{1 / q}
\end{aligned}
$$

for $1<p, q<\infty$ with $\frac{1}{p}+\frac{1}{q}=1$. Note that (2) and (3) are the endpoint inequalities of (4), which may be obtained from (4) by letting $p \rightarrow 1$ and $p \rightarrow \infty$ respectively.

As we shall show below, these inequalities can be obtained in a much simpler way without using the Grüss' type inequality. Indeed, by integration by parts, we have

$$
\frac{f(a)+f(b)}{2}(b-a)-\int_{a}^{b} f=\int_{a}^{b}\left[x-\frac{a+b}{2}\right] f^{\prime}(x) d x .
$$

Received April 24, 2001; revised July 23, 2001.

2000 Mathematics Subject Classification. 26D15.

Key words and phrases. Trapezoid inequality, Grüss' type inequality. 
Now since $\int_{a}^{b}\left[x-\frac{a+b}{2}\right] d x=0$, we see that

$$
\int_{a}^{b}\left[x-\frac{a+b}{2}\right] f^{\prime}(x) d x=\int_{a}^{b}\left[x-\frac{a+b}{2}\right]\left[f^{\prime}(x)-c\right] d x
$$

for any $c \in \mathbf{R}$. In particular, if we take $c=\frac{f(b)-f(a)}{b-a}$, then

whence

$$
\int_{a}^{b}\left[x-\frac{a+b}{2}\right] f^{\prime}(x) d x=\int_{a}^{b}\left[x-\frac{a+b}{2}\right]\left[f^{\prime}(x)-\frac{f(b)-f(a)}{b-a}\right] d x
$$

$$
\left|\int_{a}^{b}\left[x-\frac{a+b}{2}\right] f^{\prime}(x) d x\right| \leq \int_{a}^{b}\left|\left[x-\frac{a+b}{2}\right]\left[f^{\prime}(x)-\frac{f(b)-f(a)}{b-a}\right]\right| d x .
$$

The three inequalities (2)-(4) then follows immediately from this via Hölder's inequality. Notice here that $\int_{a}^{b}\left|x-\frac{a+b}{2}\right|^{p} d x=\frac{(b-a)^{p+1}}{2^{p}(p+1)}$ for $1 \leq p<\infty$, while $\sup _{x \in(a, b)}\left|x-\frac{a+b}{2}\right|=$ $\frac{b-a}{2}$.

\section{Improved Inequalities}

Since we can actually choose arbitrary values for $c$ in (5), we ask ourself now: is the choice of $c=\frac{f(b)-f(a)}{b-a}$ best possible? Note that the average value $c=\frac{f(b)-f(a)}{b-a}$ comes out automatically when one estimates the left hand side of (5) via the Grüss' type inequality (1). From our direct proof, however, we see that there is no obligation for us to use this value. Hence it is natural to ask whether we can improve the three trapezoid inequalities by choosing a different value for $c$.

An obvious way to improve them is, of course, by choosing $c=c_{0}$ such that $\sup _{x \in(a, b)}$ $\left|f^{\prime}(x)-c\right|$ or $\left[\int_{a}^{b}\left|f^{\prime}(x)-c\right|^{q} d x\right]^{1 / q}$, for $1 \leq q<\infty$, is minimized at $c_{0}$. In the case where $f^{\prime}$ is bounded, for instance, the existence of such $c_{0}$ is guaranteed by the continuity of the above expression in $c$.

Let us just consider the two endpoint inequalities here (we leave the intermediate inequality to the reader to investigate):

$$
\begin{aligned}
& \left|\frac{f(a)+f(b)}{2}-\frac{1}{b-a} \int_{a}^{b} f\right| \leq \frac{b-a}{4} \sup _{x \in(a, b)}\left|f^{\prime}(x)-c\right| \\
& \left|\frac{f(a)+f(b)}{2}-\frac{1}{b-a} \int_{a}^{b} f\right| \leq \frac{1}{2} \int_{a}^{b}\left|f^{\prime}(x)-c\right| d x
\end{aligned}
$$

where $c \in \mathbf{R}$. The following examples indicate that the choice of $c=\frac{f(b)-f(a)}{b-a}$, both in (6) and (7), is not best possible.

Example 1. Let $f(x)=x^{3}, x \in[0,2]$. Then $f^{\prime}(x)=3 x^{2} \in(0,12), x \in(0,2)$. If $c=$ $\frac{f(2)-f(0)}{2-0}=4$, then $f^{\prime}(x)-c=3 x^{2}-4 \in(-4,8), x \in(0,2)$, and hence $\sup _{x \in(0,2)}\left|f^{\prime}(x)-c\right|$ 
$=\sup _{x \in(0,2)}\left|3 x^{2}-4\right|=8$. However, if we choose $c=6$ instead of 4 , we will get $\sup _{x \in(0,2)}\left|f^{\prime}(x)-c\right|=\sup _{x \in(0,2)}\left|3 x^{2}-6\right|=6$. The value $c=6$ is clearly the one that minimizes $\sup _{x \in(0,2)}\left|f^{\prime}(x)-c\right|$.

Example 2. Again, let $f(x)=x^{3}, x \in[0,2]$. Then, as before, $f^{\prime}(x)=3 x^{2}$, $x \in(0,2)$. If $c=\frac{f(2)-f(0)}{2-0}=4$, then we have $\int_{0}^{2}\left|3 x^{2}-4\right| d x=\frac{32}{9} \sqrt{3}$. By elementary calculus, one may check that the expression $\int_{0}^{2}\left|3 x^{2}-c\right| d x$ is minimized when $c=3$. Here $\int_{0}^{2}\left|3 x^{2}-3\right| d x=6$.

Assuming that $f^{\prime}$ is bounded, one may observe that $c=\frac{1}{2}\left[\inf _{x \in(a, b)} f^{\prime}(x)+\sup _{x \in(a, b)}\right.$ $\left.f^{\prime}(x)\right]$ will minimize $\sup _{x \in(a, b)}\left|f^{\prime}(x)-c\right|$. With this choice of $c$, we have the following inequality:

$$
\left|\frac{f(a)+f(b)}{2}-\frac{1}{b-a} \int_{a}^{b} f\right| \leq \frac{b-a}{8}\left[\sup _{x \in(a, b)} f^{\prime}(x)-\inf _{x \in(a, b)} f^{\prime}(x)\right],
$$

which is generally better than (2).

Meanwhile, to find the value of $c$ that minimizes $\int_{a}^{b}\left|f^{\prime}(x)-c\right| d x$ requires further assumption on $f^{\prime}$. In particular, if $f^{\prime}(x) \leq f^{\prime}\left(\frac{a+b}{2}\right) \leq f^{\prime}(y)$ or $f^{\prime}(x) \geq f^{\prime}\left(\frac{a+b}{2}\right) \geq f^{\prime}(y)$ for $a<x<\frac{a+b}{2}<y<b$ [e.g. if $f^{\prime}$ is monotone on $(a, b)$ ], then we have the following improvement of (3):

$$
\left|\frac{f(a)+f(b)}{2}-\frac{1}{b-a} \int_{a}^{b} f\right| \leq \frac{1}{2} \int_{a}^{b}\left|f^{\prime}(x)-f^{\prime}\left(\frac{a+b}{2}\right)\right| d x
$$

This inequality is best possible in the sense that if we replace $f^{\prime}\left(\frac{a+b}{2}\right)$ by a different value, then we will obtain a worse estimate. Indeed, if we alter it by $\delta \neq 0$, then we will gain $\epsilon_{1}=(b-a)|\delta| / 2$ on one sub-interval and lose $\epsilon_{2} \leq(b-a)|\delta| / 2$ on another sub-interval, so that in total our estimate increases by $\epsilon_{1}-\epsilon_{2}$, which is nonnegative.

\section{Concluding Remarks}

The classical Grüss' inequality says that if $\phi, \psi:[a, b] \rightarrow \mathbf{R}$ are integrable and bounded, with $k \leq \phi(x) \leq K$ and $m \leq \psi(x) \leq M$ for all $x \in[a, b]$, then

$$
\left|\frac{1}{b-a} \int_{a}^{b} \phi \psi-\left[\frac{1}{b-a} \int_{a}^{b} \phi\right]\left[\frac{1}{b-a} \int_{a}^{b} \psi\right]\right| \leq \frac{1}{4}[K-k][M-m]
$$

(see [2], p. 296). If we take $\phi(x)=x-\frac{a+b}{2}$ and $\psi(x)=f^{\prime}(x)$, then we obtain

$$
\left|\frac{f(a)+f(b)}{2}-\frac{1}{b-a} \int_{a}^{b} f\right| \leq \frac{b-a}{4}\left[\sup _{x \in(a, b)} f^{\prime}(x)-\inf _{x \in(a, b)} f^{\prime}(x)\right]
$$

which is worse than (8) [and is no better than (2) either]. 
Meanwhile, by calculating the right-hand side of (9), the inequality (9) can be simplified to

$$
\left|\frac{f(a)+f(b)}{2}-\frac{1}{b-a} \int_{a}^{b} f\right| \leq\left|\frac{f(a)+f(b)}{2}-f\left(\frac{a+b}{2}\right)\right| .
$$

In the case where $f^{\prime}(x) \leq f^{\prime}\left(\frac{a+b}{2}\right) \leq f^{\prime}(y)$ for $a<x<\frac{a+b}{2}<y<b$ [e.g. when $f^{\prime}$ is increasing on $(a, b)]$, we obtain

$$
f\left(\frac{a+b}{2}\right) \leq \frac{1}{b-a} \int_{a}^{b} f \leq \frac{f(a)+f(b)}{2},
$$

which is the classical Hadamard's inequality (see [2], p. 10).

As in [1], the improved inequalities (8) and (9) may be applied to some special means (e.g. arithmetic, geometric, or logarithmic means). We leave the computation to the reader.

\section{Acknowledgements}

This work was carried out during a visit to the School of Mathematics, UNSW, Sydney, in 2000/2001, under an Australia-Indonesia Merdeka Fellowship funded by the Australian Government through the Department of Education, Training and Youth Affairs and promoted through Australia Education International. The author would also like to thank the referee for useful remarks on the earlier version of this note.

\section{References}

[1] S. S. Dragomir and A. Mcandrew, On trapezoid inequality via a Grüss' type result and applications, Tamkang J. Math. 31(2000), 193-201.

[2] D. S. Mitrinović, J. E. Pěcarić and A. M. Fink, Classical and New Inequalities in Analysis, Kluwer Academic Publishers, Dordrecht, 1993.

Department of Mathematics, Bandung Institute of Technology, Bandung 40132, Indonesia

E-mail: hgunawan@dns.math.itb.ac.id 